\title{
Dipylidium caninum
}

National Cancer Institute

\section{Source}

National Cancer Institute. Dipylidium caninum. NCI Thesaurus. Code C122285.

A species of tapeworms in the family Dipylidiidae. The definitive host for D. caninum is the dog, with intermediate hosts of fleas and chewing lice. Humans can become infected by ingesting an infected flea. 